\title{
Research and experiment of a novel flower transplanting device using hybrid-driven mechanism
}

\author{
Xiong Zhao ${ }^{1,2}$, Xiaoshu Zhang ${ }^{1}$, Qingpei $\mathrm{Wu}^{1}$, Li Dai ${ }^{2}$, Jianneng Chen ${ }^{1 *}$ \\ (1. Faculty of Mechanical Engineering and Automation, Zhejiang Sci-Tech University, Hangzhou 310018, China; \\ 2. Key Laboratory of Zhejiang Transplanting Equipment Technology, Hangzhou 310018, China)
}

\begin{abstract}
Aiming at decreasing the component complexity and cost of flower transplanting machine, an integrated transplanting method for picking and planting flower seedlings was proposed, and a hybrid-driven five-bar parallel mechanism was designed. A "beak-shaped" trajectory was designed for integrated transplanting requirements, and meantime, either the posture requirements of transplanting claw were determined. Based on the transplanting trajectory of the mechanism, a corresponding mathematical model for solving the link parameters was established, and then the five-bar mechanism was divided into two bar groups, optimization was conducted in two steps based on genetic algorithm and NSGA-II algorithm. Consequently, the optimal solution of the hybrid-driven five-bar parallel mechanism for flower seedling transplanting was obtained. Compared with similar designs, the trajectory displacement of the proposed mechanism is larger in the condition of smaller link size, which indicates that the mechanism can effectively decrease the machine size. The real-time controllable motor angular acceleration fluctuation is smaller and the commutation times are less, which has the advantage of reducing the difficulty of the mechanism control system. Subsequently, the correctness of the design method is verified by kinematics simulation. Finally, the synchronous linkage motion control methods of the two motors were designed, a transplanting experiment of the prototype was carried out, the picking success rate had reached $90 \%-93.4 \%$ and transplanting success rate was $80.5 \%-86.9 \%$ during experiment, which showed that the integrated operation of picking and planting flower seedlings can be realized by the proposed mechanism.
\end{abstract}

Keywords: hybrid-driven mechanism, mechanism optimization, integrated operation of picking and planting, synchronous linkage motion control

DOI: $10.25165 /$ j.ijabe.20201302.5187

Citation: Zhao X, Zhang X S, Wu Q P, Dai L, Chen J N. Research and experiment of a novel flower transplanting device using hybrid-driven mechanism. Int J Agric \& Biol Eng, 2020; 13(2): 92-100.

\section{Introduction}

The flower industry has become an emerging industry, in which the technology for flower transplanting reduces the growth cycle, helps to ensure uniform crop quality and increases production and income ${ }^{[1-3]}$. Countries with high degree of agricultural mechanization have developed some equipment for transplanting flowers. Using a puma560 robot as a carrier, KUTZ $\mathrm{L} \mathrm{J}$ designed a special seedling gripper based on $\mathrm{CAD}$, this gripper installed on the robot could achieve the transplanting of bedding plants but the efficiency was too low ${ }^{[4]}$. TING $\mathrm{K} \mathrm{C}$ took the Adept One SCARA robot as the ontology and designed a transplanting robot with four degrees of freedom ${ }^{[5]}$. This robot used an end-effector with a visual sensor and a force sensor to complete the transplanting operation, but the equipment was expensive. The Dutch company VISSER developed a transplanter,

\section{Received date: 2019-06-01 Accepted date: 2020-02-22}

Biographies: Xiong Zhao, Associate Professor, research interests: mechanism design, non-circular gear transmissions, and agricultural machinery. Email: zhaoxiong@zstu.edu.cn; Xiaoshu Zhang, Master candidate, research interests: agricultural machinery, Email: 2495264516@qq.com; Qingpei Wu, MS, research interests: agricultural machinery, Email: 1032705530@qq.com; Li Dai, Associate Professor, research interests: agricultural machinery, Email: dl@ zstu.edu.cn.

*Corresponding author: Jianneng Chen, Professor, research interests: machine design, non-circular gear transmissions and control, and agricultural machinery. Faculty of Mechanical Engineering and Automation, Zhejiang Sci-Tech University, Hangzhou 310018, China. Tel: +86- 13065701536, Email: jiannengchen@zstu.edu.cn. which has high automation level and efficiency but poor versatility. To achieve the integration of picking and planting, it is necessary to cooperate with Mayer-its special pot supply machine ${ }^{[6]}$. The Urbinati RW series transplanters designed in Italy can work with single and multiple rows of grippers and has a transplanting efficiency reaching up to 36000 plants per hour. Its disadvantages include an insufficient gripper force of the seedling claws, an easy leakage of seedlings and a high equipment maintenance cost. CMP, a Canadian automation solution supplier, developed a plant transplanting pipeline device, which covers a large area and requires professional customization, additionally, the equipment set is expensive. The seedling claws in the above equipment insert the seedling vertically in the vertical direction, without considering the damage to seedling leaves, and the suitable variety of seedlings for operation is limited.

The transplanting of flower seedlings belongs to the category of plug seedling transplantation, but the flower transplantation machines and equipment in China are not perfect. Chinese flower planting enterprises are generally smaller in scale and need transplanting equipment with simple components and low cost. The process is mainly based on manual transplanting, which has a large demand for labour. Hu applied the Delta robot to flower seedling transplanting ${ }^{[7]}$. Its seedling claw can move in three directions, but the robot does not meet the requirement that the seedling claw should change its posture. Ryu developed a robotic transplanter for bedding plants, but its transplanting efficiency is not high $^{[8]}$. The intelligent transplanting machine for flower seedlings developed by Feng is equipped with an industrial 
camera $^{[9]}$. Through visual identification, deficient and diseased seedlings are removed, which can improve the quality of transplanting; but it also failed to popularize because of use-cost. Jin utilized a double-crank five-bar mechanism to satisfy the function of seedling picking in vegetables, but it is difficult to achieve more complicated trajectory ${ }^{[10]}$. Some research results of other scholars are also not applicable to the integrated operation of seedling picking and planting ${ }^{[11-14]}$.

A hybrid-driven mechanism is an innovative whose scope is between a traditional machinery and a modern robot. Compared with traditional machinery, it has not only the advantages of stable operation, convenient manufacture and low cost but also the characteristics of a full servo-driven mechanism that can achieve complex motion rules and flexible outputs ${ }^{[15,16]}$. The author had achieved the function of flower plug seedling picking by the hybrid-driven five-bar mechanism ${ }^{[17]}$. Based on hybrid-driven mechanism, this paper aimed to realize integrated operation of picking and planting flower seedlings, the kinematics rules, structure parameters and motor control parameters of the mechanism were optimized according to the new functional requirements. This research achieves the required kinematics trajectory, at the same time, compared with the mechanism which only realized the function of seedling picking, the link parameters in this paper are more compact and the motor motion stability is better.

\section{Principle of mechanism and design of transplanting trajectory}

\subsection{Principle of mechanism}

The hybrid-driven mechanism for planting flower into flowerpots uses the planar, full-hinge, five-bar mechanism for the motion carrier, as shown in Figure 1. $L_{A E}$ is the frame, $L_{A B}$ and $L_{E D}$ are driving links, and $L_{A B}$ is driven by a real-time controllable motor $M_{1}$, which adjusts the movement of the mechanism in real time. $L_{E D}$ is a crank driven by a constant speed motor $M_{2}$. Bar group $\mathrm{CDF}$ is the entirety, with the transplanting arm mounted on CDF. The T-shaped transplanting arm takes $B, C$ and $F$ as its vertices, and its mass is relatively large; if driven by a real-time controllable motor, the inertial force of the transplanting arm causes an unstable operation of the mechanism when speed fluctuation or motor commutation occurs. The arm is relatively stable when driven by a constant speed motor. $L_{B C}$ is a coupler,

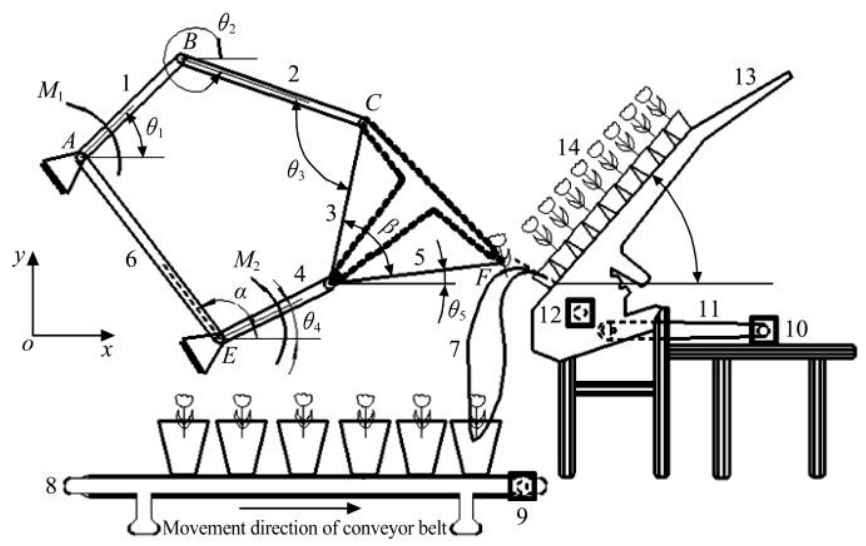

$\begin{array}{llll}\text { 1. Real-time controllable side link } L_{A B} & \text { 2. Coupler } L_{B C} & \text { 3. } L_{C D} & \text { 4. Crank } L_{E D}\end{array}$ 5. $L_{D F} \quad$ 6. Frame $L_{A E} \quad$ 7. Transplanting trajectory 8 . Conveyor belt 9. Conveyor motor 10. Seedling feeding motor 11. Transmission chain 12. Line feed motor 13. Seedling box 14. Flower seedlings

Figure 1 Schematic diagram of mechanism and experimental platform point $C$ of the transplanting arm is hinged with coupler $L_{B C}$, point $D$ is hinged with crank $L_{E D}$, and point $F$ is the execution end of the mechanism. The combined motion of two driving links $L_{A B}$ and $L_{E D}$ forms the trajectory of execution end point $F$.

\subsection{Design of transplanting trajectory}

Through the experimental platform model of Figure 1, according to the agronomic requirements and the parameters of the experimental platform, the corresponding transplanting trajectory was designed. The following are required for the trajectory of flower seedling transplanting:

(1) The section of seedling picking process. As shown in Figures 1 and $2 \mathrm{a}$, the angle $\delta$ between the seedling box and the horizontal plane can be adjusted between $45^{\circ}$ and $60^{\circ}$, so the angle $\gamma$ between the seedling and the horizontal plane is between $135^{\circ}$ and $150^{\circ}$. The swing angle $\eta$ of the seedling claw should be within $10^{\circ}$ in the seedling picking process and the width of the beak should be less than $5 \mathrm{~mm}$, which prevent the seedling claw from interfering with the seedling pot. Flower seedlings are usually 4-6 cm high and have 3-5 leaves. To prevent damage to the leaves and stems of the seedlings, the seedling claw should enter the seedling pot from the lower left corner of the seedlings, and gradually rotate to parallel to the stem of the seedling in the process of entering the pot.

(2) The section of seedling transporting. The seedling claw should clamp the seedlings and not fall down during the process of downward transporting. The height $h$ of the transplanting trajectory should be as high as possible to prevent interference between the flowerpot and the bottom of the experimental platform.

(3) The section of the seedling claw entering and exiting the flowerpot. The trajectory should be as vertical as possible to the surface of the flowerpot, with the seedlings oriented vertically when the seedling claw plants them. The width $w$ at $60 \mathrm{~mm}$ (about half of the flowerpot's depth) in the vertical direction from the lowest point of the trajectory should be less than $120 \mathrm{~mm}$ (diameter of the upper surface of the flowerpot) to prevent the seedling claw from interfering with the flowerpot.

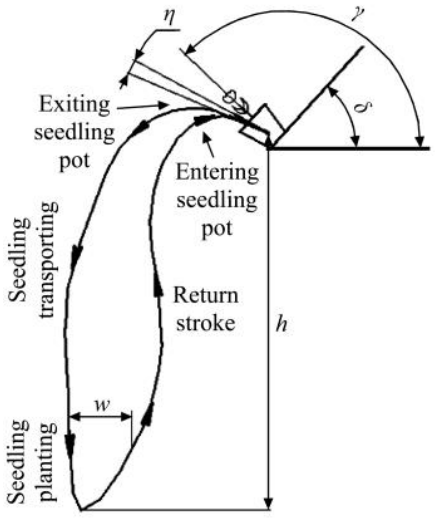

a. Trajectory function analysis diagram

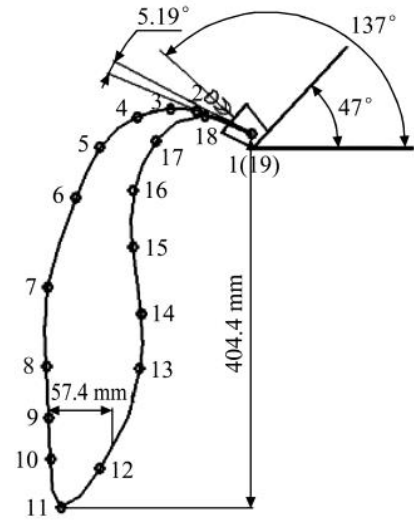

b. Shape control points analysis diagram
Figure 2 Schematic diagram of trajectory

According to the above requirements, in CAD, 19 trajectory shape control points (in which the first and last points are the same) are selected, and these points are connected by a spline curve to obtain a "beak-shaped" trajectory. The transplanting height $h$ is initially set as $404.4 \mathrm{~mm}$ initially, the bottom width is $57.4 \mathrm{~mm}$, and the width of the beak is $3 \mathrm{~mm}$, the swing angle $\eta$ of seedling claw is $5.19^{\circ}$, the angle $\gamma$ is $137^{\circ}$, as shown in Figure $2 \mathrm{~b}$. The coordinates of 19 control points on the trajectory are shown in Table 1. 
Table 1 Coordinates of trajectory shape control points

\begin{tabular}{cc||cc}
\hline Number & Coordinate & Number & Coordinate \\
\hline 1 & $(250.5,450.6)$ & 11 & $(47.1,47.2)$ \\
2 & $(193.1,475.3)$ & 12 & $(88.7,89.4)$ \\
3 & $(163.8,477.1)$ & 12 & $(130.7,197.6)$ \\
4 & $(128.6,468.5)$ & 14 & $(133.1,258.1)$ \\
5 & $(88.4,436.2)$ & 15 & $(123.6,328.2)$ \\
6 & $(62.9,381.6)$ & 16 & $(124.5,390.5)$ \\
7 & $(31.8,284.8)$ & 17 & $(149.7,443.9)$ \\
8 & $(31.9,200)$ & 18 & $(200.1,469.3)$ \\
9 & $(34.2,143.7)$ & 19 & $(250.5,450.6)$ \\
10 & $(35.8,98.9)$ & & \\
\hline
\end{tabular}

\section{Mathematical model of mechanism optimization}

\subsection{Mathematical model of mechanism}

Disassembling the five-bar mechanism from the hinge centre point $C$ into two bar groups, namely, the bar group $E D F$ and the bar group $A B C$ and using the coordinates of the 19 shape control points that point $F$ traverses in a period, the mechanical parameters in Figure 1 and the kinematic inverse solution method of the open-chain $2 \mathrm{R}$ bar group ${ }^{[18]}$, the lengths $l_{4}$ and $l_{5}$ of link $L_{E D}$ and link $L_{D F}$ can be obtained.

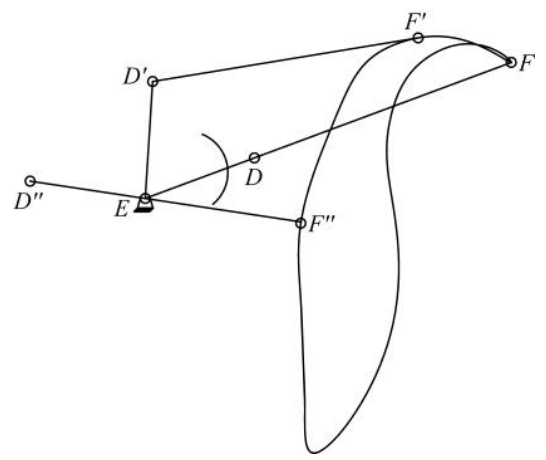

Figure 3 Mathematical model building principle

It is known that in one cycle the mechanism end point $F$ needs to pass through 19 trajectory shape control points, as shown in the Figure 3. During the rotation, the crank $L_{E D}$ and the connecting bar $L_{D F}$ have two collinear positions, corresponding to the furthest distance and the closest distance between the point $E$ and the trajectory respectively, so that the length of the crank $L_{E D}$ and the connecting rod $L_{D F}$ can be obtained. $x_{E}, y_{E}, x_{F}, y_{F}$ are the $x$ and $y$ coordinates of the hinge points $E$ and $F$. In this paper, the unit of link length and hinge point coordinate is $\mathrm{mm}$, degrees unit are in radians.

$\left\{\begin{array}{l}l_{4}=\frac{\max \left(\sqrt{\left(y_{F}-y_{E}\right)^{2}+\left(x_{F}-x_{E}\right)^{2}}\right)-\min \left(\sqrt{\left(y_{F}-y_{E}\right)^{2}+\left(x_{F}-x_{E}\right)^{2}}\right)}{2} \\ l_{5}=\frac{\max \left(\sqrt{\left(y_{F}-y_{E}\right)^{2}+\left(x_{F}-x_{E}\right)^{2}}\right)+\min \left(\sqrt{\left(y_{F}-y_{E}\right)^{2}+\left(x_{F}-x_{E}\right)^{2}}\right)}{2}\end{array}\right.$

According to the geometric relationship, the angular displacement $\theta_{4}$ of crank $L_{E D}$ can be obtained.

$\theta_{4}=\tan ^{-1}\left(\frac{y_{F}-y_{E}}{x_{F}-x_{E}}\right) \pm \cos ^{-1}\left(\frac{l_{4}^{2}-l_{5}^{2}+\left[\left(y_{F}-y_{E}\right)^{2}+\left(x_{F}-x_{E}\right)^{2}\right]}{2 \cdot l_{4} \cdot \sqrt{\left(y_{F}-y_{E}\right)^{2}+\left(x_{F}-x_{E}\right)^{2}}}\right)$

The sign in the equation is defined as follows: positive when the alphabetical order of the $\triangle E D F$ three-vertex $E D F$ is clockwise and negative when its alphabetical order is anticlockwise.

The coordinate of point $D$ in one cycle can be obtained by the following equation:

$$
\left\{\begin{array}{l}
x_{D}=x_{E}+l_{4} \cdot \cos \theta_{4} \\
y_{D}=y_{E}+l_{4} \cdot \sin \theta_{4}
\end{array}\right.
$$

After acquiring the coordinate of the hinge centre point $D$, the angular displacement $\theta_{5}$ of link $L_{D F}$ can be obtained as follows:

$$
\theta_{5}=\tan ^{-1}\left(\frac{y_{F}-y_{D}}{x_{F}-x_{D}}\right)
$$

The coordinates of point $C$ in one cycle are the following:

$$
\left\{\begin{array}{l}
x_{C}=x_{D}+l_{3} \cdot \cos \left(\theta_{5}+\beta\right) \\
y_{C}=y_{D}+l_{3} \cdot \sin \left(\theta_{5}+\beta\right)
\end{array}\right.
$$

$\beta$ is the angle between $L_{C D}$ and $L_{D F}$ in Figure 1.

The following are the coordinates of point $A$ :

$$
\left\{\begin{array}{l}
x_{A}=x_{E}+l_{6} \cdot \cos \alpha \\
y_{A}=y_{E}+l_{6} \cdot \sin \alpha
\end{array}\right.
$$

where, $\alpha$ is the angle between frame $L_{A E}$ and the horizontal plane, as shown in Figure 1.

Since the bar group $E D F$ and the bar group $A B C$ have the same mathematical model. After obtaining the coordinates of points $A$ and $C$, according to solution method bar group $E D F$ the parameters in the bar group $A B C$ are solved as follows, the lengths $l_{1}$ and $l_{2}$ of link $L_{A B}$ and link $L_{B C}$ can be obtained.

$\left\{\begin{array}{l}l_{1}=\frac{\max \left(\sqrt{\left(y_{C}-y_{A}\right)^{2}+\left(x_{C}-x_{A}\right)^{2}}\right)-\min \left(\sqrt{\left(y_{C}-y_{A}\right)^{2}+\left(x_{C}-x_{A}\right)^{2}}\right)}{2} \\ l_{2}=\frac{\max \left(\sqrt{\left(y_{C}-y_{A}\right)^{2}+\left(x_{C}-x_{A}\right)^{2}}\right)+\min \left(\sqrt{\left(y_{C}-y_{A}\right)^{2}+\left(x_{C}-x_{A}\right)^{2}}\right)}{2}\end{array}\right.$

$$
\theta_{1}=\tan ^{-1}\left(\frac{y_{C}-y_{A}}{x_{C}-x_{A}}\right) \pm \cos ^{-1}\left(\frac{l_{1}^{2}-l_{2}^{2}+\left[\left(y_{C}-y_{A}\right)^{2}+\left(x_{C}-x_{A}\right)^{2}\right]}{2 \cdot l_{1} \cdot \sqrt{\left(y_{C}-y_{A}\right)^{2}+\left(x_{C}-x_{A}\right)^{2}}}\right)
$$

The sign in the equation is defined as follows: positive when the alphabetical order of the $\triangle A B C$ three-vertex $A B C$ is clockwise and negative when its alphabetical order is anticlockwise.

\subsection{Mechanism parameters optimization}

Through the analysis of the second section, the variables involved in the optimization are $\left\{\left(x_{E}, y_{E}\right), l_{3}, \beta, l_{6}, \alpha\right\}$. The optimization process is divided into two steps: the optimization of bar group $E D F$ and that of bar group $A B C$.

\subsubsection{Optimization of bar group EDF}

This step optimizes the coordinate of point $E$. With point $E$ and the 19 shape control points on the trajectory, $l_{4}$ and $l_{5}$ can be solved, and their corresponding angular displacements $\theta_{4}$ and $\theta_{5}$ are obtained.

Let

$$
k=\frac{\theta_{4}(1)-\theta_{4}(\max (i))}{1-\max (i)} \quad(i=1 \ldots 19)
$$

Objective function:

$$
f\left(x_{E}, y_{E}\right)=\min \sum_{i=1}^{19} a b s\left(k i+\theta_{4}(1)-k-\theta_{4}(i)\right)^{2}
$$

where, $k$ is the slope of the straight line formed by connecting the first and last data points on the curve with the ordinal number of the 19 shape control trajectory points as abscissa and the 19 angular displacement $\theta_{4}$ values of crank $L_{E D}$ corresponding to the 19 shape control trajectory points as longitudinal coordinate. The objective function of optimization is to make the crank angular displacement $\theta_{4}$ corresponding to the 19 shape control points as close as possible to the straight line with slope $k$. Since the crank $L_{E D}$ rotates at a constant speed, the rotation angle is the same in unit time; therefore, the crank angle displacement $\theta_{4}$ corresponding to point $F$ at any position should be on a straight line with a certain slope. 
Design variables: $(E x, E y)$

Constraints:

$$
\theta_{4}(i+1)-\theta_{4}(i)>0 \quad(i=1 \ldots 19)
$$

$$
y_{E}-47.2>l_{4}
$$

Condition (c1) renders the crank angular displacement monotonous, and condition (c2) prevents interference between the crank $L_{E D}$ and the flowerpot due to an excessive length of the crank $L_{E D}$. The number 47.2 is the ordinate of point 11 (the lowest point of the trajectory).

The upper and lower limits of the design variable:

$$
\left\{\begin{array}{l}
l u=[-200,0] \\
l p=[150,500]
\end{array}\right.
$$

Control parameters of the genetic algorithm: choice: roulette; mutation probability: 0.01 ; crossover probability: 0.8 ; the total number of individuals in the population: 100; the maximum number of iterations: 400.

Because a genetic algorithm has certain randomness, after multiple operations of the optimized program, the following results can be obtained: $\left(x_{E}, y_{E}\right)=(-130,310)$. According to Equation (1), the length $l_{4}$ of $L_{E D}$ is calculated to be $121 \mathrm{~mm}$, and the length $l_{5}$ of $L_{D F}$ is $275 \mathrm{~mm}$. The genetic iteration process is shown in Figure 4.

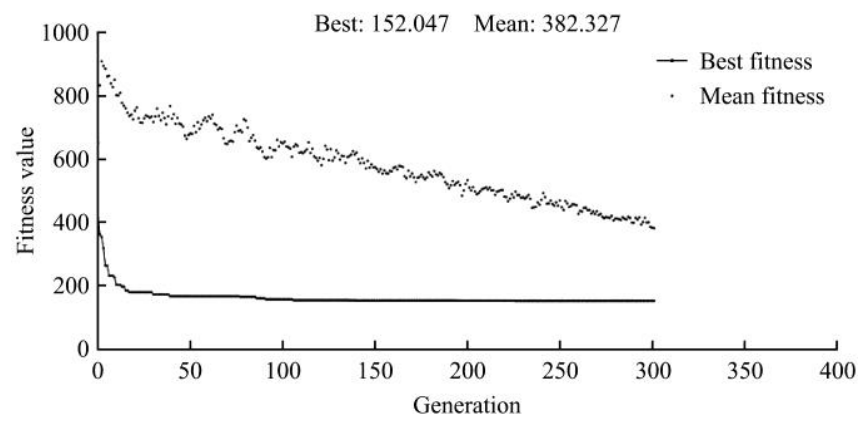

Figure 4 Genetic iteration process

Using Equations (2) and (4), the angular displacement $\theta_{4}$ values of crank $L_{E D}$ corresponding to 19 shape control coordinate points are obtained. The angular displacement $\theta_{5}$ values corresponding to 19 angular displacement $\theta_{4}$ values of crank are also obtained. The solid line in Figure 4 shows a curve with 19 angular displacement $\theta_{4}$ values of crank $L_{E D}$ on the abscissa and 19 angular displacement $\theta_{5}$ values of $L_{D F}$ on the ordinate.

Only 19 data points can be calculated initially, to obtain more data points and smooth angular displacement curves, a cubic non-uniform B - spline is adopted, 360 data points and the interpolated and densified curve is shown by the dotted line in Figure 5.

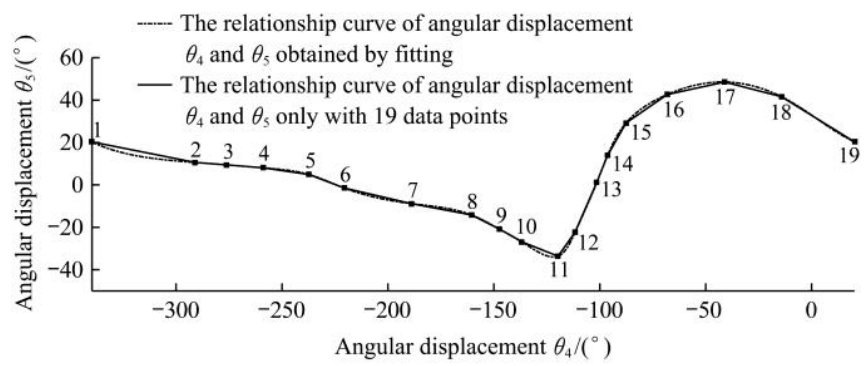

Figure 5 Relationship Curve of the angular displacement $\theta_{4}$ and $\theta_{5}$

After fitting, the angular displacement $\theta_{4}$ of crank $L_{E D}$ is shown by the dotted line in Figure 6. Crank $L_{E D}$ is driven by a constant-speed motor, and the connecting line between any two angular displacement data points of crank $L_{E D}$ should be on a straight line with a certain slope, thus linearizing the angular displacement $\theta_{4}$ to form a straight line with a certain slope, on which the difference between the ordinate values of two adjacent data points is $1^{\circ}$, as shown by the solid line in Figure 6 . According to the relationship between $\theta_{4}$ and $\theta_{5}$ as shown by the dotted line in Figure 6, the new $\theta_{5}$ value corresponding to new $\theta_{4}$ is calculated by linear interpolation, and the Figure 7 curve is obtained. The forward calculation is performed via the new angular displacement data in Figure 7 and $(E x, E y), l_{4}, l_{5}$ to obtain final trajectory of the mechanism.

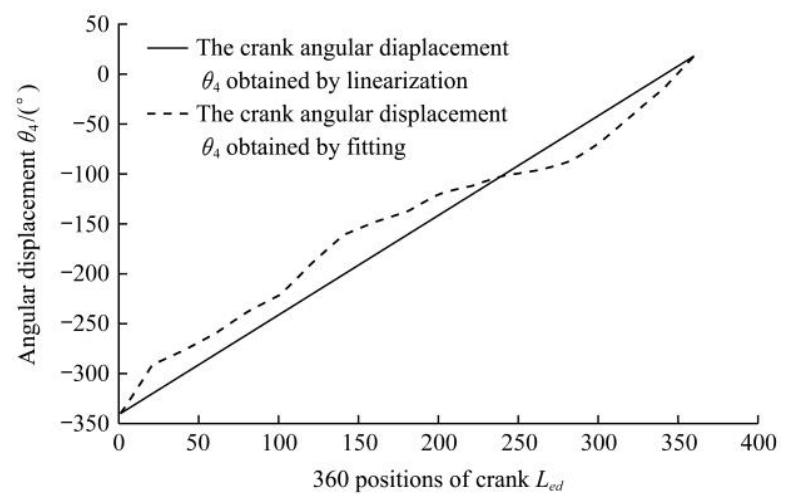

Figure 6 Crank angular displacement $\theta_{4}$ obtained by fitting and linearization

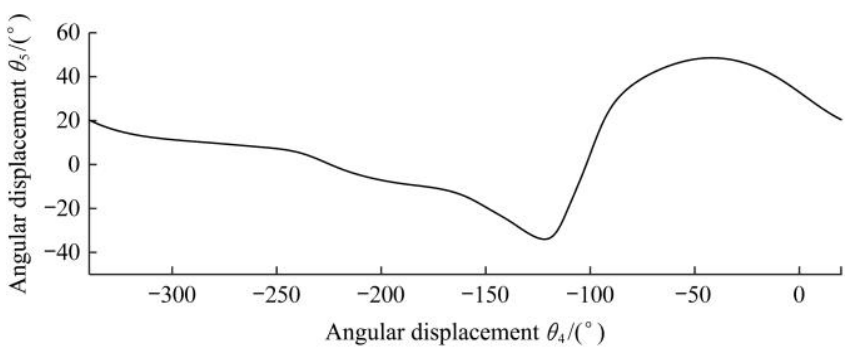

Figure 7 New relationship curve of $\theta_{4}$ and $\theta_{5}$ obtained by linear interpolation

The final trajectory curve is compared with the initial trajectory curve as designed in Figure $2 b$. The shape and the height of the trajectory are consistent, and all the indicators meet the design requirements.

3.2.2 Optimization of bar group ABC

There are two optimization goals for this step: 1) Try to make the sum of the ratio of $L_{A B}$ to $L_{B C}$ and the ratio of $L_{E D}$ to $L_{C D}$ smaller so that the centre of gravity of the mechanism will be relatively stable. 2)The maximum acceleration of the real-time controllable side link $L_{A B}$ under the working condition that the seedling transplanting efficiency is 60 plants/min is as small as possible, because the real-time controllable side link $L_{A B}$ is driven by the real-time controllable motor, which is usually a servomotor with an angular acceleration limit. If the maximum angular acceleration is too large, the motor shaft cannot respond accurately, which affects the control accuracy.

The NSGA-II optimization algorithm is relatively effective for multi-objective optimization, during which an important problem is the assignment of adaptive values to individuals. The present paper adopts the Pareto sorting method. In the treatment of constraints, variable penalty is adopted, and the variable penalty coefficient is adjusted according to the degree of constraint violation and the number of iterations of the genetic algorithm.

Objective function: 


$$
\left\{\begin{array}{l}
f_{1}=\min \left(\left(\frac{L_{A B}}{L_{B C}}\right)+\left(\frac{L_{E D}}{L_{C D}}\right)\right) \\
f_{2}=\min \left(\max \left(\operatorname{abs}\left(\ddot{\theta}_{1}\left(0^{\circ}-360^{\circ}\right)\right)\right)\right)
\end{array}\right.
$$

Design variables: $\left\{l_{6}, l_{3}, \alpha, \beta\right\}$.

The upper and lower limits of the design variable:

$$
\left\{\begin{array}{l}
l u=[0,0,0,0,0] \\
l p=\left[300,300,2 \pi, \frac{5}{9} \pi\right]
\end{array}\right.
$$

Constraints:

(c1) According to the necessary conditions for crank existence and the conditions for avoiding singular configurations:

$$
\begin{gathered}
\max \left(\operatorname{sqrt}\left(\left(x_{B}-x_{D}\right)^{2}+\left(y_{B}-y_{D}\right)^{2}\right)\right)<L_{B C}+L_{C D} \\
\operatorname{abs}\left(L_{B C}-L_{C D}\right)<\min \left(\operatorname{sqrt}\left(\left(x_{B}-x_{D}\right)^{2}+\left(y_{B}-y_{D}\right)^{2}\right)\right)
\end{gathered}
$$

(c2) Limit of maximum angular acceleration of servomotor:

$$
\ddot{\theta}\left(0^{\circ}-360^{\circ}\right) \leq 100 \pi \mathrm{rad} / \mathrm{s}^{2}
$$

According to the technical manual of the Delta servomotor, the servomotor shaft rises from $0 \mathrm{r} / \mathrm{min}$ to a maximum speed of $3000 \mathrm{r} / \mathrm{min}$; the minimum time required is $1 \mathrm{~s}$. After calculation, the limit value of the motor shaft angular acceleration is $100 \pi$ $\mathrm{rad} / \mathrm{s}^{2}$.

(c3) Limit of real-time controllable side link $L_{A B}$ commutation times:

$$
\operatorname{crests}\left(\theta_{1}\right)+\operatorname{troughs}\left(\theta_{1}\right) \leq 3
$$

The servomotor shaft causes the phenomenon of "pulse cancellation" during commutation, which leads to motor shaft failing to reach the expected position. So that the commutation times of the control motor are as few as possible in the optimization, the mathematical relation expresses that the number of crests and troughs of the angular displacement curve of real-time controllable side link $L_{A B}$ be as few as possible during the optimization process, making the number of commutations less than or equal to three.

Control parameters of the genetic algorithm: The population comprises 150 individuals, which are encoded by real numbers. The total crossover probability is 0.8 , using an arithmetic crossover operator and a mixed crossover operator. The total mutation probability is 0.2. Multiple uniform mutation and multiple non-uniform mutation operators are adopted. The maximum number of iterations is 300 . The Pareto front is shown in Figure 8 .

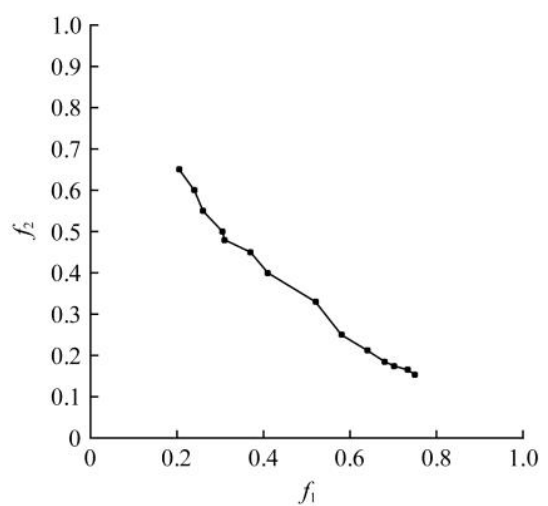

Figure 8 Pareto front

The Pareto solution sets obtained by the optimization are shown in Table 2.

Compared with other solutions, the angular displacement function curve of the two driving links solved by the third group $(117.8,152.2,2.198$ and 1.38) of solutions has better linearity on both sides of the crest and the trough in the angular displacement curve of two driving links. The obtained length of the links makes the structure more compact, and the angle parameter is more suitable. The following are obtained from Equation (7): $l_{1}=$ $94.6 \mathrm{~mm}$ and $l_{2}=112.1 \mathrm{~mm}$.

Table 2 Pareto solution sets

\begin{tabular}{cccc}
\hline$l_{6} / \mathrm{mm}$ & $l_{3} / \mathrm{mm}$ & $\alpha / \mathrm{rad}$ & $\beta / \mathrm{rad}$ \\
\hline 129.2 & 118.6 & 2.512 & 1.099 \\
138.7 & 113.3 & 0.680 & 1.491 \\
117.8 & 152.2 & 2.198 & 1.383 \\
40.1 & 146.8 & 2.471 & 1.302 \\
96.7 & 133.4 & 0.063 & 1.275 \\
61.8 & 145.7 & 1.984 & 1.156 \\
173.6 & 108.3 & 0.488 & 1.434 \\
111.8 & 123.5 & 0.652 & 1.283 \\
157.2 & 123.9 & 2.391 & 1.021 \\
265.6 & 176.01 & 0.175 & 1.179 \\
135.1 & 108.4 & 0.741 & 1.374 \\
69.2 & 128.9 & 2.375 & 1.290 \\
143.3 & 139.4 & 2.510 & 1.373 \\
227.9 & 219.6 & 0.364 & 1.296 \\
\hline
\end{tabular}

Finally, all mechanical parameters of the mechanism are obtained: $\left\{(E x, E y), l_{3}, \beta, l_{6}, \alpha\right\}=\{(-130,310), 152.2 \mathrm{~mm}, 1.383$ radian, $117.8 \mathrm{~mm}, 2.198$ radian $\}, l_{1}=94.6 \mathrm{~mm}, l_{2}=112.1 \mathrm{~mm}$, $l_{4}=121 \mathrm{~mm}, l_{5}=275 \mathrm{~mm}$. At the same time, the angular displacement curve of two driving links is obtained, as shown by the dotted line in Figure 9.

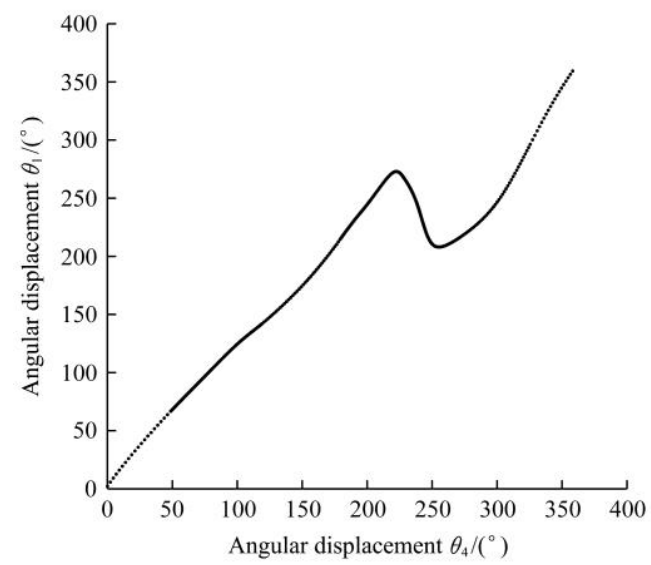

Figure 9 Angular displacement curves of two driving links

\subsection{Analysis of optimization results}

3.3.1 Analysis of the angular displacement curves of two driving links

The abscissa in Figure 10 is the angle displacement $\theta_{4}$ of crank $L_{E D}$, and the ordinate is the angular displacement $\theta_{1}$ of the real-time controllable side link $L_{A B}$. The angular displacement curve indicated by the solid line is the optimization result of reference 17 , which has two crests and two troughs; and the real-time controllable side link $L_{A B}$ commutates four times in one cycle. The angular displacement curve represented by the dotted line is the optimization result of the present paper. One crest and one trough occur, and the number of commutations is two, two less than that reported in reference 17 . It can be seen that adding the constant constraint of motor fluctuation in the new optimization model is very beneficial to the design results.

3.3.2 Analysis of kinematical characteristics

In Figure 10, the solid-line trajectory is the trajectory of reference 17 , and the dotted-line trajectory is that defined in the 
present paper.

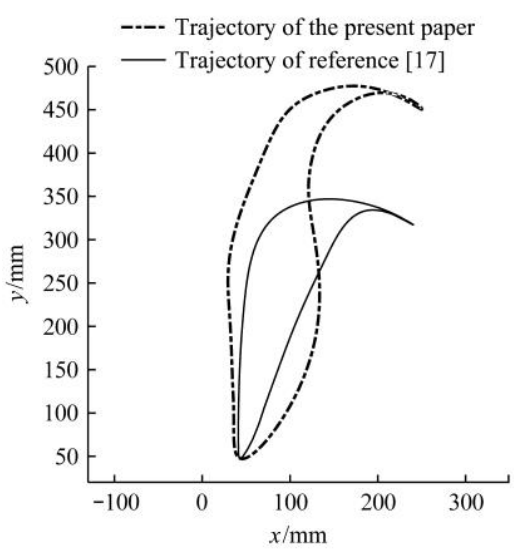

Figure 10 Comparison of trajectories

The transplanting height $h$ of the solid trajectory is $265 \mathrm{~mm}$, the bottom width $w$ of the trajectory is $20 \mathrm{~mm}$, and the width of the beak is $2 \mathrm{~mm}$. The angle of the seedling claw is $150^{\circ}$ when it touches the pot, the swinging angle $\eta$ of the seedling claw in picking seedling process is $6.27^{\circ}$ in reference 17 .

The transplanting height $h$ of the dotted-line track is $404.4 \mathrm{~mm}$, the bottom width $w$ of the trajectory is $57.41 \mathrm{~mm}$, and the width of the beak is $3 \mathrm{~mm}$. The angle of the seedling claw is $152^{\circ}$ when it touches the pot, the swinging angle $\eta$ of the seedling claw in picking seedling process is $5.19^{\circ}$ in the present paper.

Compared with the dotted-line trajectory, the solid-line trajectory is not large enough for the transplanting height. However, in the actual experiment, insufficient height will lead to interference between the flowerpot and the bottom of the experimental platform, and the flowerpot will be pushed down by the supporting element at the bottom of the experimental platform, which will affect the transplanting work. The higher transplanting height will avoid the above situation. In addition, at the "neck" of the trajectory, the dotted trajectory is thinner than the solid trajectory, and interference with the seedling box is unlikely.

3.3.3 Analysis of the angular acceleration curves of real-time controllable motor

The curves in Figure 11 are the angular acceleration of the real-time controllable side link under the working condition that the seedling picking efficiency is 60 seedlings $/ \mathrm{min}$. The dotted line is the optimization result of reference 17 , showing that the angular acceleration of the real-time controllable motor fluctuates greatly and that 27 angular acceleration data points exceed the motor angular acceleration limit, causing the motor shaft connected to $L_{A B}$ to respond inaccurately at these points, thereby failing to reach the expected position. This failure affects the trajectory of the execution end point $F$, mainly because the physical characteristics of the motor are not taken into consideration in the optimization. The solid line in the Figure 11 is the optimization result of the present paper; although the angular acceleration also fluctuates, the maximum angular acceleration does not exceed the motor angular acceleration limit, and the acceleration value at the fluctuation is greatly reduced. This result occurs because the constraint of the angular acceleration limit of the motor is added to the optimization and the maximum acceleration is kept as small as possible in the objective function.

3.3.4 Analysis of mechanical parameters

The right side of Figure 12 is the motion simulation of reference 17 , and the left side is the motion simulation of the present paper. The second row of Table 3 is the mechanical parameters of reference 17, and the third row of Table 3 is the mechanical parameters of the present paper. From Figure 12 and Table 3, it can be seen intuitively that the mechanical parameters of the present paper make the structure more compact and the transplanting displacement higher.

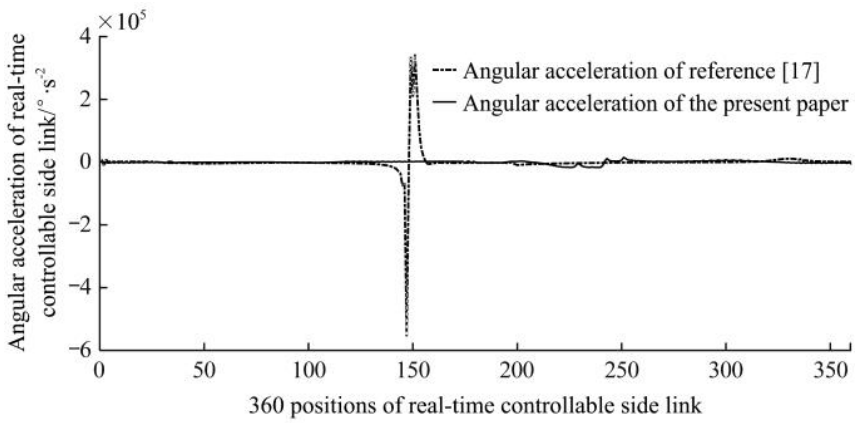

Figure 11 Comparison of the angular acceleration curves of real-time controllable motor

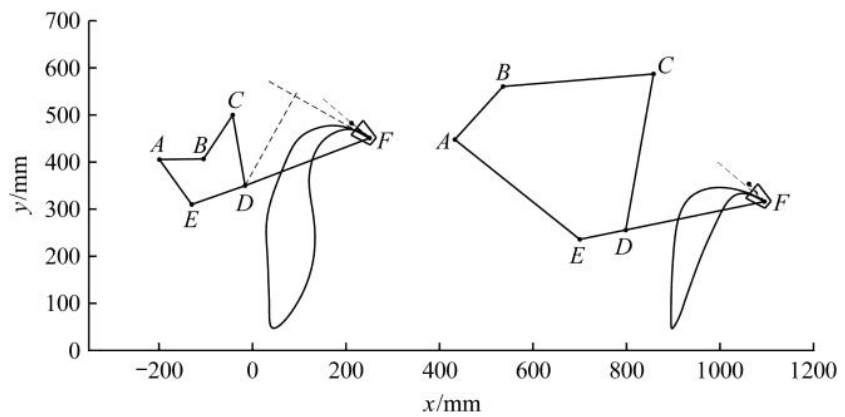

Figure 12 Comparison diagram of the motion simulation

Table 3 Comparison of mechanical parameters

\begin{tabular}{ccc}
\hline Parameter & Result of reference [17] & Result of the present paper \\
\hline$l_{1}(\mathrm{~mm})$ & 152 & 94.6 \\
$l_{2}(\mathrm{~mm})$ & 324.6 & 112.1 \\
$l_{3}(\mathrm{~mm})$ & 336.6 & 152.2 \\
$l_{4}(\mathrm{~mm})$ & 100.4 & 121 \\
$l_{5}(\mathrm{~mm})$ & 302.6 & 275 \\
$l_{6}(\mathrm{~mm})$ & 341 & 117.8 \\
$\alpha(\mathrm{rad})$ & 2.478 & 2.198 \\
$\beta(\mathrm{rad})$ & 1.192 & 1.383 \\
\hline
\end{tabular}

\section{Structural design of the mechanism and kinematical} simulation

\subsection{Mechanism control strategy}

The model of the mechanism is designed according to the optimized mechanical parameters, as shown in Figure 13a. The mechanism is imported into Adams, and the angular displacement function of the two-driving links is imported into Adams as the driving function of the two-driving links; the mechanism is simulated after adding corresponding constraints, forces and kinematic pairs. The simulation interface is shown in Figure 13b, and a comparison of the simulation and theoretical trajectories is shown in Figure 14.

In Figure 14, the theoretical trajectory is marked 1, and the simulation trajectory is marked 2. In the simulation interface of Figure 13b, a small tail can be seen in bottom of trajectory. In the structural design, when the seedling planting is finished, the shifting fork slides into a groove on the cam attached to crank $L_{E D}$, and the seedling claw retracts under the action of the spring, thus shortening the length of $l_{5}$. Simultaneously, on the return stroke, 
the shifting fork is initially in the groove on the cam, the seedling claw is still in a retracted state, and the trajectory of the return stroke narrows. Before the seedling picking action, the cam doesn't push seedling claw out, the neck of the track is still narrower than that of the theoretical trajectory. When the seedling claw moves to the apex of the trajectory's beak, $l_{5}$ returns to its original length; then, the shifting fork is pressed tightly by the cam, so that the other part trajectories of the seedling claw are coincide with the original trajectory.

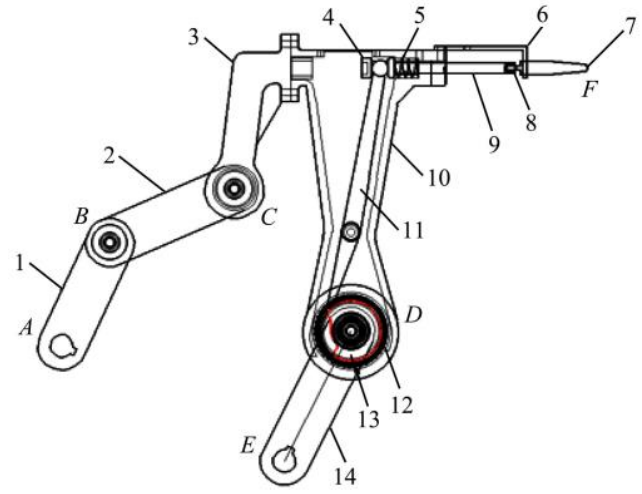

a. Schematic of the mechanism

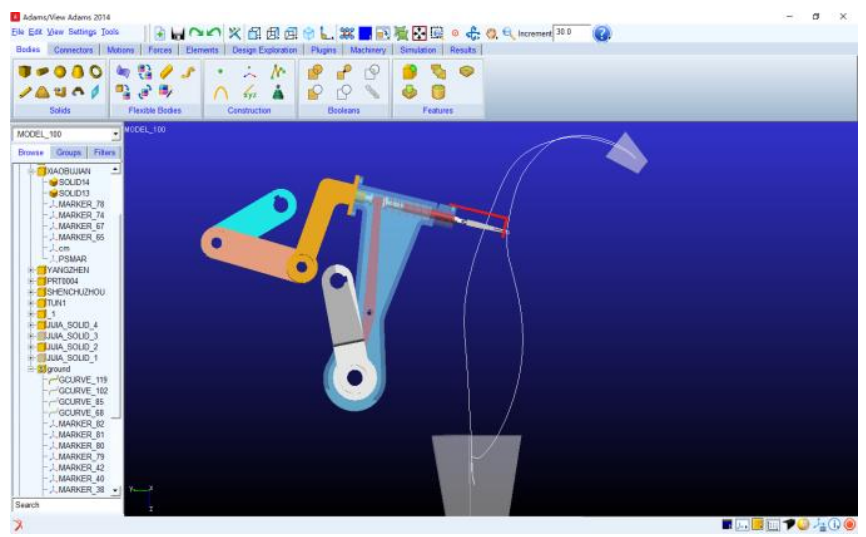

b. Simulation interface of mechanism

$\begin{array}{lll}\text { 1. Real-time controllable side link } L_{A B} & 2 \text {. Coupler } L_{B C} & 3 \text {. End cap of the }\end{array}$ transplanting arm shell 4. Shift fork block 5. Spring 6. Guide slot of seedling claw 7. Seedling claw 8. Connecting plate of seedling claw 9. Seedling pushing bar 10. Transplanting arm shell 11. Shift fork 12. Ball bearing 13. Cam attached to crank $L_{E D}$ 14. Crank $L_{E D}$

Figure 13 Schematic and the simulation interface of the mechanism
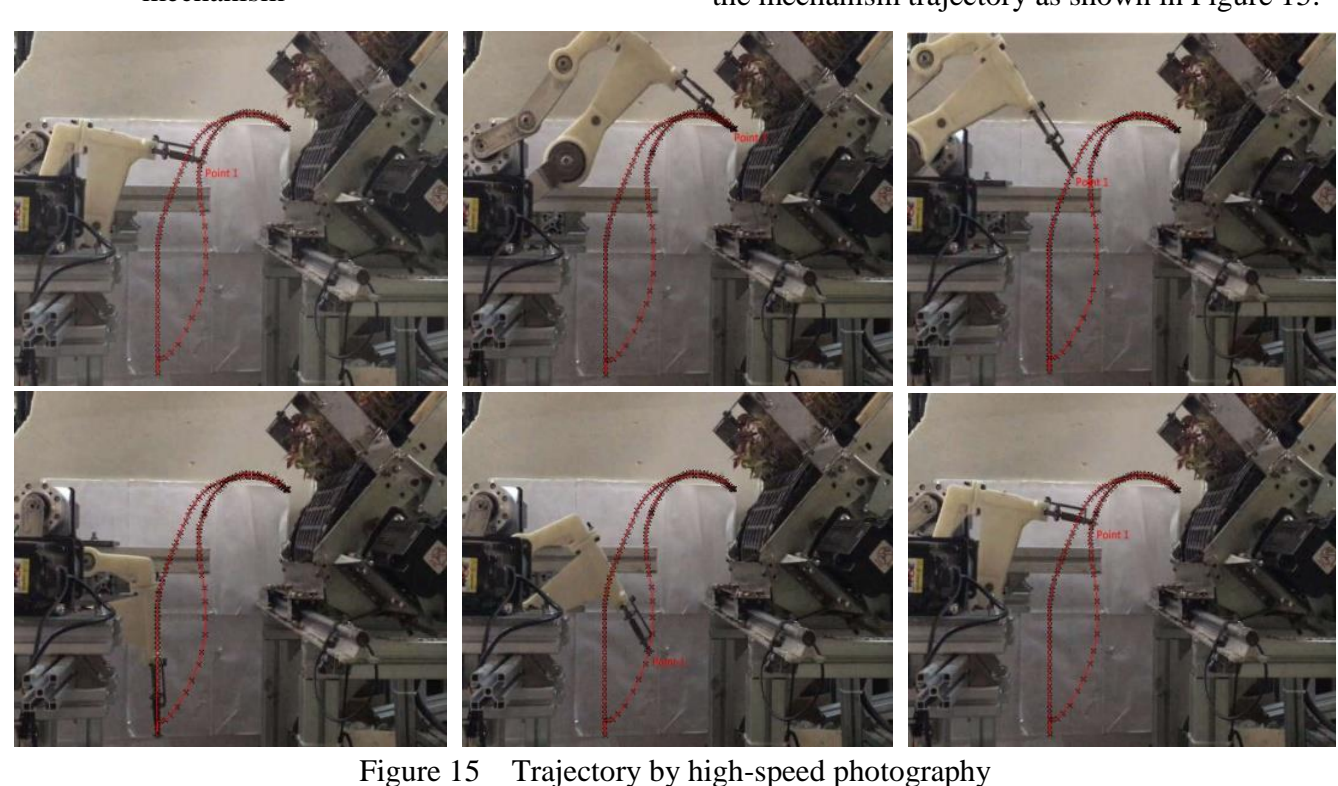

Figure 15 Trajectory by high-speed photography

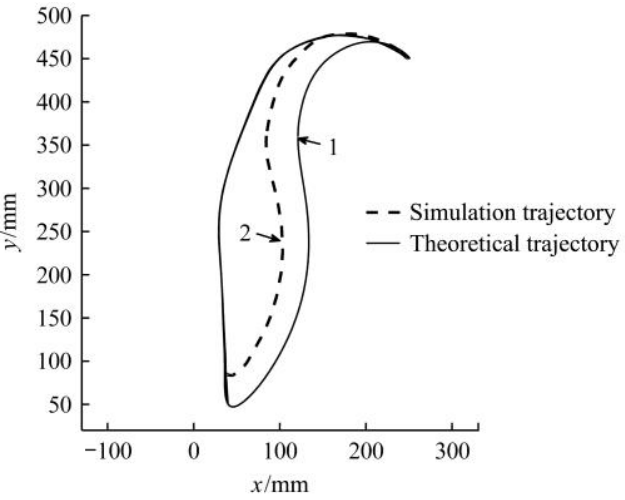

Figure 14 Comparison of theoretical and simulation trajectories

To ensure the experimental effect and the motion accuracy of the mechanism, the present study uses a servomotor to simulate the uniform motion of a constant-speed motor. Because of their strict positional correspondence during movement, the two motors belong to a multi-shaft linkage control. Therefore, the DDA linear interpolation algorithm is adopted for motor control. The angular displacement function curve of the two-driving links indicated by the dotted line in Figure 5 is divided into 360 segments by taking one segment on the curve corresponding to 1 degree of crank rotation. These small segments are fitted by the DDA linear interpolation algorithm, and the two motors of the mechanism move according to the fitted function curve. The execution end point $F$ of the mechanism will sequentially pass through the points on the trajectory to ensure the accuracy of the trajectory.

The controller adopts an STM32ZET6 minimum system board and effectuates synchronous control of the two motors by writing a DDA algorithm program. The controller's general-purpose timer encoder module is applied to analyse the servomotor's encoder feedback pulse. The pulse information is fed back to the computer through an RS232 communication protocol, and the pulse is recorded and compared with the pulse sent by the controller. If any position error occurs, pulse compensation is performed.

\subsection{Transplanting experiment of mechanism}

A no-load test was conducted before the seedlings transplanting experiment. A motion video of the mechanism is imported into the high-speed photography analysis software Blaster's MAS to detect the trajectory of the mechanism, obtaining the mechanism trajectory as shown in Figure 15. 
The trajectory obtained in the no-load test is largely consistent with the trajectory of the Adams simulation in Figure 13b, which proves the accuracy of the structural design and the control system.

The mechanism prototype is as shown in the Figure 16a. The mechanism test bench shown in Figure $16 \mathrm{~b}$ is constructed of aluminium profiles, and the motors are installed on the test bench connected with two original moving parts of the mechanism.

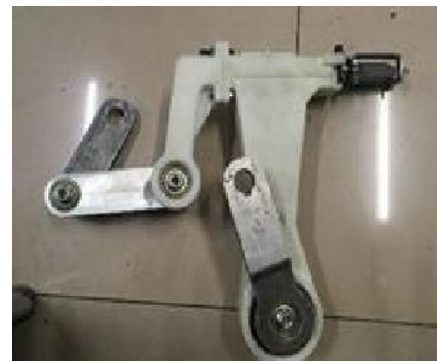

a. Prototype

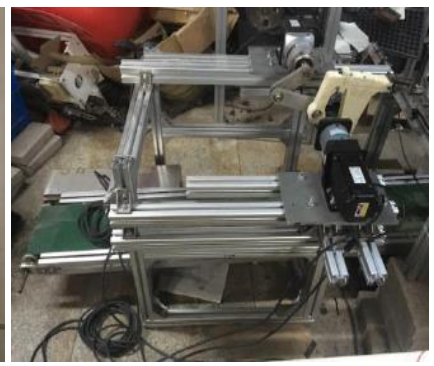

b. Test bench
Figure 16 Prototype and test bench

Houttuynia cordata seedlings cultivated for $25 \mathrm{~d}$ were chosen as the experimental materials, the height of the seedlings is between $80 \mathrm{~mm}$ and $100 \mathrm{~mm}$, the rate of seedling emergence is about $90 \%$, and the water content of seedling bowl is about $50 \%$. The seedling tray is 16 rows and 8 columns. The upper surface of square holes in the tray that each accommodate one seedling is $31 \mathrm{~mm} \times 31 \mathrm{~mm}$, their lower surface is a square of $18 \mathrm{~mm} \times 18 \mathrm{~mm}$, and their depth is $44 \mathrm{~mm}$. The upper surface diameter of the flowerpots into which seedlings are transplanted is $120 \mathrm{~mm}$, the diameter of their lower surface is $100 \mathrm{~mm}$, and their depth is $115 \mathrm{~mm}$. The H. cordata seedlings, seedling tray and flowerpot are shown in Figure 17a. The seedling picking and planting postures of the mechanism are shown in Figure 17b, and the transplanting effect of the mechanism is shown in Figure 17c.

The transplanting efficiency of the mechanism was 60 seedlings per minute in the transplanting experiment. The average seedling picking distance is $40 \mathrm{~mm}$, and the average seedling planting distance is $50 \mathrm{~mm}$. The success rate of picking seedlings was $90 \%, 93.4 \%$ and $92.3 \%$. The success rate of planting seedlings was $85.8 \%, 86.9 \%$ and $80.5 \%$. The data for the transplanting experiments are shown in Table 4. Judging from the experimental results, the mechanism realized integrated operation of seedling picking and planting for flower, the success rate of seedling picking is relatively ideal, and the success rate of seedling planting is slightly lower than that of seedling picking. Through comprehensive analysis, there are two reasons for the results, one factor is the density of the soil, when the soil in the flowerpot is too dense, the substrate of the seedling is destroyed during planting, which lead to plant failure. Another factor is the coordination of the movement of the flowerpot feeding device and transplanting mechanism, if flower seedlings cannot plant in the central area of the flowerpot, seedling claw may push the pot over, which increasing the number of failures. The elimination of these two factors will improve the success rate of seedling planting.
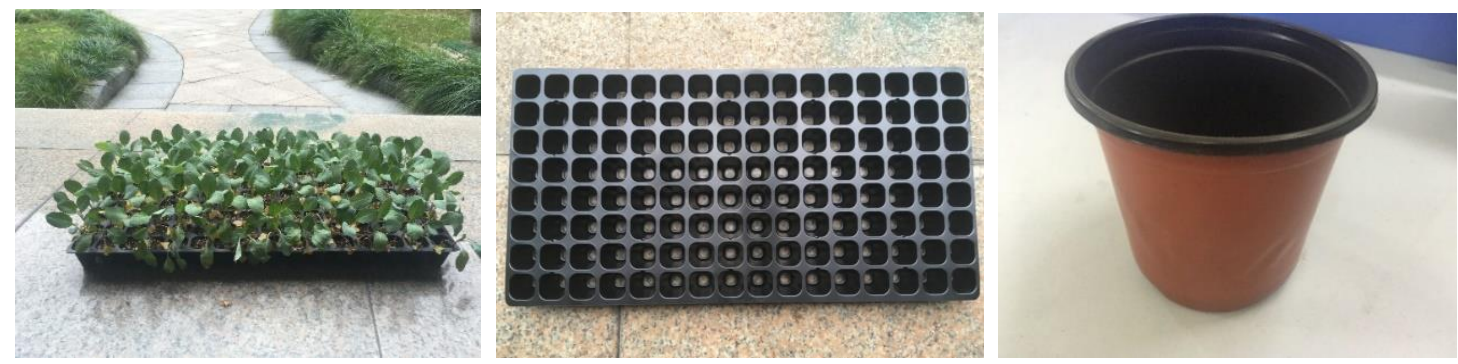

a. Pictures of seedlings, seedling tray and flowerpot

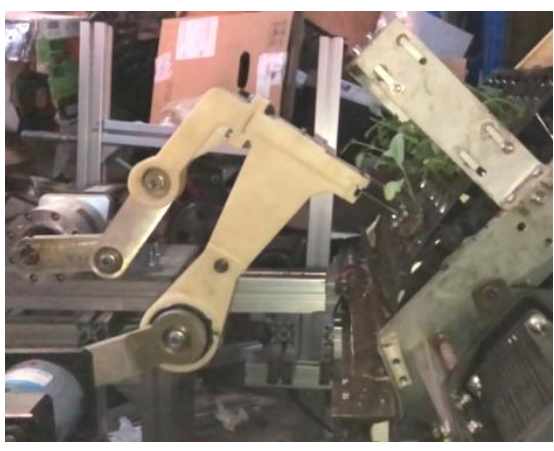

b. Postures for picking and planting seedlings

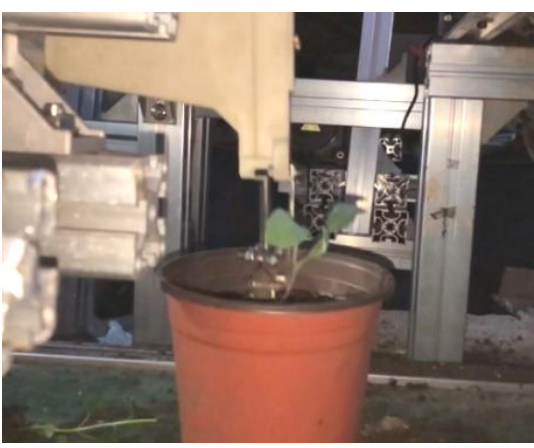

Figure 17 Pictures related to the experiment

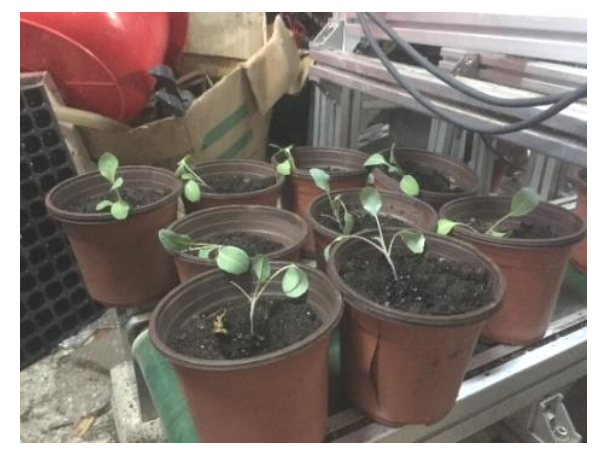

c. Transplanting effect of mechanism

Table 4 Transplanting experiment

\begin{tabular}{|c|c|c|c|c|c|c|c|}
\hline $\begin{array}{l}\text { Seedling tray } \\
\text { number }\end{array}$ & $\begin{array}{l}\text { Number of pots, each } \\
\text { with one seedling }\end{array}$ & $\begin{array}{c}\text { Number of } \\
\text { deficient seedlings }\end{array}$ & $\begin{array}{l}\text { Actual number of } \\
\text { seedlings }\end{array}$ & $\begin{array}{c}\text { Number of } \\
\text { successfully picking }\end{array}$ & $\begin{array}{c}\text { Number of } \\
\text { successfully planting }\end{array}$ & $\begin{array}{c}\text { Success rate of } \\
\text { picking seedlings/\% }\end{array}$ & $\begin{array}{c}\text { Success rate of } \\
\text { planting seedlings } / \%\end{array}$ \\
\hline 1 & 128 & 8 & 120 & 108 & 103 & 90.0 & 85.8 \\
\hline 3 & 128 & 10 & 118 & 109 & 95 & 92.3 & 80.5 \\
\hline
\end{tabular}

\section{Conclusions}

The ideal flower seedling transplanting trajectory was defined according to the agronomic requirements. With optimized design, a set of improved mechanical parameters was selected: $\{(E x, E y)$, $\left.l_{3}, \beta, l_{6}, \alpha\right\}=\{(-130,310), 152.2 \mathrm{~mm}, 1.383$ radian, $117.8 \mathrm{~mm}$, 2.198 radian $\}, l_{1}=94.6 \mathrm{~mm}, l_{2}=112.1 \mathrm{~mm}, l_{4}=121 \mathrm{~mm}$ and $l_{5}=$ $275 \mathrm{~mm}$. Additionally, the angular displacement function curve of the two driving links is also obtained. The structure of the mechanism obtained by this set of parameters is relatively compact, 
the angular acceleration fluctuation of the real-time controllable side link is reduced, and the linearity of two driving links' angular displacement curve has improved on both sides of the crest and the trough.

The experiment was carried out by transplanting the Houttuynia cordata seedlings as an example. The transplanting trajectory of the mechanism obtained by the high-speed photography software is consistent with the virtual simulation trajectory, which verifies the correctness of the mechanism design and motor control mode. In the transplanting experiment, the seedling claw can accurately pick the flower seedlings out from the seedling pot and its postures meet the requirements in the picking process. During the planting process, the flower seedlings can be vertically planted into the flowerpot, the integrated operation of picking and planting flower seedlings was realized by the proposed mechanism. Transplanting experiment showed that the mechanism could achieve a picking success rate of 90\%-93.4\% and a transplanting success rate of $80.5 \%-86.9 \%$. The research could provide a novel and simple mechanism for flower transplanting machine.

\section{Acknowledgements}

The research work was financially supported by the National Natural Science Foundation of China (Grant No. 51775512, 51975536); Key research projects of Zhejiang Province (Grant No. 2018C02046); Project funded by China Postdoctoral Science Foundation; Basic public welfare research projects of Zhejiang Province (Grant No. LGN19E050002, LGN20E050006) and Basic Scientific Research Foundation of Zhejiang Sci-Tech University.

\section{[References]}

[1] Zhang L H, Qiu L H, Tian S B. Progress in the research of manipulator of transplanting potted tray seedlings. Agricultural Science \& Technology and Equipment, 2009; 10(5): 28-31.

[2] Han C J, Zhang X J, Yang W Z, Yin W Q. Present status and analysis of dry-land auto-transplanting seedling technique. Journal of Agricultural Mechanization Research, 2011; 33(11): 238-240. (in Chinese)

[3] Gao G H, Zhang S, Liu Q. Research status of automatic transplanting technology of potted flowers. Agricultural Engineering Technology, 2018;
22(2): 16-17. (in Chinese)

[4] Kutz L J, Craven J B. Robotic transplanting of bedding plants Transaction of the ASAE, 1987; 30(3): 0586-0590.

[5] Ting K C, Giacomell G A, Shen S J, Kabala W P. Robot workcell for transplanting of seedlings part II end-effector development. ASABE, 1990; 33(3): 1013-1018.

[6] Hu J P, Yan X Y, Ma J, Qi C H, Francis K, Mao H P. Dimensional synthesis and kinematics simulation of a high-speed plug seedling transplanting robot. Computers \& Electronics in Agriculture, 2014; 107(3): 64-72.

[7] Pic-O-Mat PF Transplanter. Avaiable at: http://www.visser.eu/plug-transplanters/pic-o-mat-pf/. Accessed on [2014-06-16].

[8] Ryu K H, Kim G, Han J S. Development of a robotic transplanter for bedding plants. J. Agric. Eng. Res., 2001; 78(2): 141-146.

[9] Feng Q C, Wang X. Design and Simulation of Automatic Transplanter for Flower Seedling. Journal of Agricultural Mechanization Research, 2013; (11): 250-252. (in Chinese)

[10] Jin X, Li D Y, Ma H, Ji J T, Zhao K X, Pang J. Development of single row automatic transplanting device for potted vegetable seedlings. Int $\mathbf{J}$ Agric \& Biol Eng, 2018; 11(3): 67-75.

[11] Yi S J, Liu Y F, Wang C, Tao G X, Liu H Y, Wang R H. Experimental study on the performance of bowl-tray rice precision seeder. Int J Agric \& Biol Eng, 2014; 7(1): 17-25.

[12] Feng Q C, Zhao C J, Jiang K, Fan P F, Wang X. Design and test of tray-seedling sorting transplanter. Int J Agric \& Biol Eng, 2015; 8(2): 14-20.

[13] Prasanna Kumar G V, Raheman H. Automatic feeding mechanism of a vegetable transplanter. Int J Agric \& Biol Eng, 2012; 5(2): 20-27.

[14] Ye B L, Yi W M, Yu G H, Gao Y, Zhao X. Optimization design and test of rice plug seedling transplanting mechanism of planetary gear train with incomplete eccentric circular gear and non-circular gears. Int J Agric \& Biol Eng, 2017; 10(6): 43-55.

[15] Li C-H, Tso P-L. Experimental study on a hybrid-driven servo press using iterative learning control. International Journal of Machine Tools and Manufacture, 2008; 48(2): 209-219.

[16] Zi B, Zhu Z C, Cao J B. Design and Analysis of Hybrid-driven-based Cable Parallel Mechanism. Journal of Mechanical Engineering, 2011; 47(17): 1-8. (in Chinese)

[17] Zhao X, Cui $\mathrm{H} \mathrm{Y,} \mathrm{Dai} \mathrm{L.} \mathrm{Optimal} \mathrm{design} \mathrm{and} \mathrm{experiment} \mathrm{of}$ hybrid-driven five-bar flower potted-seedling transplanting mechanism. Transactions of the CSAE, 2017; 33(15): 34-40. (in Chinese)

[18] Zhao X, Wang C, Yang M X, Sun L, Chen J N. Reverse design and analysis of automatic seedling pick-up mechanism with non-circular gear planetary train. Transactions of the CSAE, 2015; 31(16): 30-36. (in Chinese) 